\title{
A HIGH-RESOLUTION SPECTRUM OF THE EXTREMELY METAL-RICH BULGE G DWARF OGLE-2006-BLG-265 ${ }^{1}$
}

\author{
Jennifer A. Johnson, ${ }^{2}$ Avishay Gal-Yam, ${ }^{3,4}$ Douglas C. Leonard, ${ }^{5}$ Joshua D. Simon, ${ }^{3}$ \\ ANDRZEJ UdALSKI, ${ }^{6,7}$ AND ANDREW Gould ${ }^{2,8}$ \\ Received 2006 August 30; accepted 2006 December 11; published 2007 January 9
}

\begin{abstract}
We present an $R=45,000$ Keck spectrum of the microlensed Galactic bulge G dwarf OGLE-2006-BLG-265, which has high ( 60) signal-to-noise ratio despite its short (15 minute) exposure time, because the source was magnified by $A \sim 135$. While it is very metal-rich $([\mathrm{Fe} / \mathrm{H}]=0.56)$, the higher temperature of this star compared with the luminous red giants usually measured in the bulge gives its spectrum many unblended atomic lines. We measure the abundances of 17 elements, including the first abundances for $\mathrm{S}$ and $\mathrm{Cu}$ in a bulge star. The $[\alpha / \mathrm{Fe}]$ ratios are subsolar, while the odd- $\mathrm{Z}$ elements are slightly supersolar, trends that are also seen in the more metalrich stars in the bulge and the local Galactic disk. Because the star is a dwarf, the $[\mathrm{O} / \mathrm{Fe}],[\mathrm{Na} / \mathrm{Fe}]$, and $[\mathrm{Al} / \mathrm{Fe}]$ ratios cannot be attributed to internal mixing, as is sometimes claimed for giants. Similar high-resolution spectra could be obtained for about a dozen bulge dwarf stars per year by means of well-designed target-of-opportunity observations.
\end{abstract}

Subject headings: Galaxy: abundances — Galaxy: bulge — Galaxy: evolution — gravitational lensing — stars: abundances

Online material: machine-readable table

\section{INTRODUCTION}

While high-resolution spectroscopy is the gold standard of stellar analysis, the daunting photon requirements of this technique generally limit its application to the brightest stars within a given stellar population, i.e., the giants. The one major exception is stars from the local neighborhood, where the dwarfs can partially compensate for their dimness by their greater numbers, and hence (statistically) their greater proximity. For distant systems, like globular clusters and the Galactic bulge, highresolution spectra of dwarfs would require exposure times that are at least 100-1000 times longer than those of giants, which in most cases is prohibitive.

Nevertheless, there are compelling reasons to obtain dwarf spectra in these systems. Element abundances in dwarfs and giants are not necessarily the same even when the progenitors of these stars formed from gas with identical compositions. Mixing on the giant branch can destroy some elements and dredge up others.

Measuring the surface-composition differences between dwarfs and giants in the same population is an important test of theories of stellar evolution. Moreover, even when the dwarf and giant element abundances are identical, the derived values may differ if the atmospheric models used to estimate the abundances are in error. Hence, comparing dwarf and giant spectra is also a powerful way to test atmospheric models. Driven by

\footnotetext{
${ }^{1}$ The data presented here were obtained at the W. M. Keck Observatory, which is operated as a scientific partnership among the California Institute of Technology, the University of California, and the National Aeronautics and Space Administration. The Observatory was made possible by the generous financial support of the W. M. Keck Foundation.

${ }^{2}$ Department of Astronomy, Ohio State University, Columbus, $\mathrm{OH}$; jaj@astronomy.ohio-state.edu,gould@astronomy.ohio-state.edu.

${ }^{3}$ Department of Astronomy, California Institute of Technology, Pasadena, CA; avishay@astro.caltech.edu.

${ }^{4}$ Hubble Fellow.

${ }^{5}$ Department of Astronomy, San Diego State University, San Diego, CA; leonard@sciences.sdsu.edu.

${ }^{6}$ Warsaw University Observatory, Warsaw, Poland; udalski@astrouw.edu.pl.

${ }^{7}$ Optical Gravitational Lens Experiment (OGLE).

${ }^{8}$ Microlensing Follow-Up Network $(\mu \mathrm{FUN})$.
}

these considerations, several groups (e.g., Boesgaard et al. 1998), have, by prodigious efforts, obtained high-resolution spectra of subgiant and main-sequence turnoff stars in globular clusters.

High-magnification gravitational microlensing events provide a unique opportunity to acquire such spectra. Microlensing events are rare, affecting only about $\tau \sim 10^{-6}$ Galactic bulge stars at any given time. The fraction of all events with maximum magnifications $A_{\max } \gg 1$ is only $A_{\max }^{-1}$. Nevertheless, by monitoring more than a hundred million bulge stars, the Optical Gravitational Lens Experiment (OGLE-III) ${ }^{9}$ Early Warning System (EWS; Udalski 2003) has been finding about $600 \mathrm{mi}-$ crolensing events in each of the last $4 \mathrm{yr}$, of which about a dozen per year have $A_{\max }>100$. The Microlensing Observations for Astrophysics ${ }^{10}$ collaboration has recently inaugurated the second phase of their experiment and expects to soon find a comparable number of high-magnification events.

High-magnification microlensing events remain within half their peak magnification for a time interval (Einstein 1936)

$$
t_{1 / 2}=\frac{\sqrt{12} t_{\mathrm{E}}}{A_{\max }} \sim 1 \text { day } \frac{t_{\mathrm{E}}}{30 \text { days }}\left(\frac{A_{\max }}{100}\right)^{-1}
$$

where $t_{\mathrm{E}}$ is the Einstein timescale of the event.

Hence, for typical Einstein timescales ( 30 days), high-magnification events will be observable by any given telescope at magnifications $A>50$. At substantially higher magnifications, the number of events accessible to an observatory falls as $A^{-2}$, one factor of $A^{-1}$ because the events are intrinsically rarer, and a second such factor because the chance that the event will peak near the observatory drops. Nevertheless, there is at least one example of an $A_{\max }=3000$ event, OGLE-2004-BLG-343, that peaked over the Very Large Telescopes in Chile and was basically recognized as such several hours before peak (Dong et al. 2006). Therefore, at least in principle, it would be possible

\footnotetext{
${ }^{9}$ See http://www.astrouw.edu.pl/ ogle/ogle3/ews/ews.html.

${ }^{10}$ See http://www.massey.ac.nz/ iabond/alert/alert.html.
} 


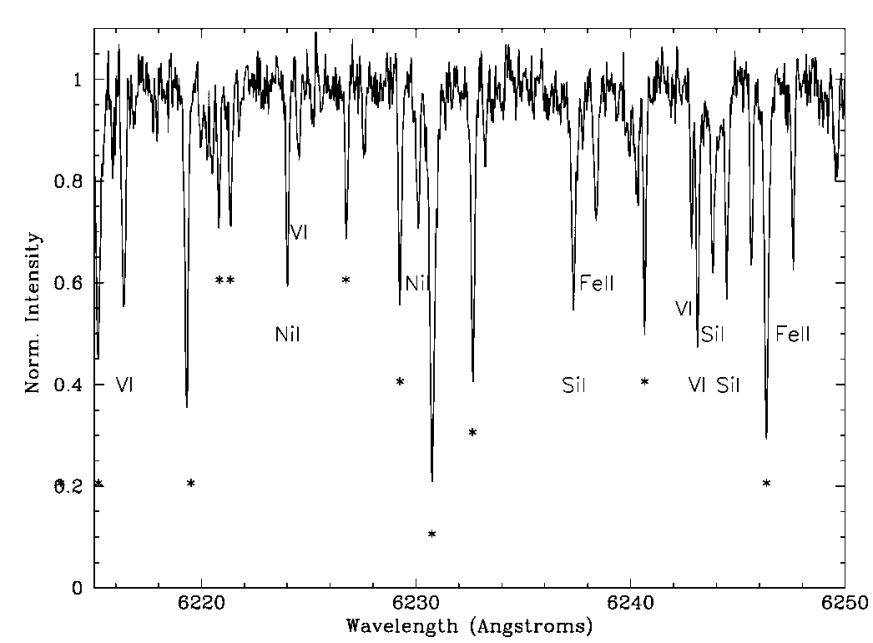

FIG. 1.-Portion of the spectrum of OGLE-2006-BLG-265. Most of the strong lines are labeled. The Fe I lines are marked with an asterisk.

to obtain of order a dozen spectra of dwarf stars in the Galactic bulge per year, each magnified by a factor $A \sim 100$, with a few at much higher magnifications.

Benetti et al. (1995) took the first spectrum of a microlensing event toward the bulge, albeit at low dispersion, but sufficient to determine the spectral type. Minniti et al. (1998) reported the possible detection of $\mathrm{Li}$ in a bulge dwarf. Cavallo et al. (2003) presented a preliminary abundance analysis for six stars with high-resolution spectra including three dwarfs magnified by factors between 2.3 and 30 . Here we report on the abundances of 17 elements in a microlensed bulge dwarf star based on high-resolution spectra, including the first measurements of $\mathrm{S}$ and $\mathrm{Cu}$.

\section{OBSERVATIONS}

OGLE-2006-BLG-265 was alerted as a probable microlensing event toward the Galactic bulge (R.A. $=18^{\mathrm{h}} 07^{\mathrm{m}} 18^{\mathrm{s}} 9$, decl. $\left.=-27^{\circ} 47^{\prime} 43^{\prime \prime} \quad[\mathrm{J} 2000] ; \quad l=3.39^{\circ}, \quad b=-3.55^{\circ}\right)$ by OGLE EWS on 2006 May 25. On June 5, OGLE issued a further alert that this would be a high-magnification event with $A>60$. Intensive photometric observations were then carried out by several collaborations, including the Microlensing Follow-Up Network $(\mu \mathrm{FUN}),{ }^{11}$ primarily with the aim of searching for planets (Mao \& Paczyński 1991; Griest \& Safizadeh 1998; Udalski et al. 2005; Gould et al. 2006). Results of that search will be presented elsewhere. The event actually peaked on June 6 (HJD 2,453,893.238) at $A_{\max } \sim 220$. Two of us (A.G.-Y., J. D. S.) happened to be at the Keck Telescope when we received the flurry of $\mu \mathrm{FUN}$ emails describing this event. We interrupted our normal program to obtain two exposures of this event, which totaled 15 minutes. The observations were carried out approximately 3.6 and $2.7 \mathrm{hr}$ before peak, when the magnification averaged $A=135$.

Using standard microlensing techniques (e.g., Yoo et al. 2004), $\mu$ FUN determined that the dereddened color and magnitude of the source were $(V-I)_{0}=0.63 \pm 0.05, I_{0}=$ $18.11 \pm 0.10$. OGLE independently found similar values. The uncertainties are due to possible differential reddening between the microlensed source and the red clump, which is assumed to have the same $(V-I)_{0}=1.00$ as the local Hipparcos clump. If (as seems likely) the source lies at approximately the

\footnotetext{
${ }^{11}$ See http://www.astronomy.ohio-state.edu/ microfun/.
}

galactocentric distance, then its absolute magnitude is $M_{V}=$ 4.3 and its radius is $1.2 R_{\odot}$. That is, it is a solar-type star.

We observed OGLE-2006-BLG-265 using the High Resolution Echelle Spectrometer (HIRES; Vogt. et al. 1994) mounted on the Keck I 10 m telescope on 2006 June 6 with a $600 \mathrm{~s}$ exposure ending at 14:07:11 UT and a $300 \mathrm{~s}$ second exposure ending at 14:49:53 UT. These spectra were obtained under good conditions (clear sky, $<1$ " seeing). We used a $0.81^{\prime \prime} \times 3.5^{\prime \prime}$ slit, yielding a resolution of 45,000 . We binned by $\times 2$ in the spatial direction to increase the signal-to-noise ratio $(\mathrm{S} / \mathrm{N})$. The data were reduced using the MAKEE package (Barlow 2002) and additional IRAF ${ }^{12}$ scripts we have developed. The reduction included flat-fielding using internal flats, wavelength calibrations using Th-Ar arcs, and MAKEE extraction using the trace of a bright calibration star. The two spectra were combined with SCOMBINE in IRAF. We also observed a rapidly rotating hot star for telluric line removal and a radial velocity (RV) standard. We focused our analysis on the data from 5355 to $8365 \AA$, where the $\mathrm{S} / \mathrm{N}$ was the highest. There are small gaps in the spectrum, but only in the reddest orders. The $\mathrm{S} / \mathrm{N}$ per resolution element is $\sim 60$ over most of the analyzed spectrum. By cross-correlating (with FXCOR) the spectrum of OGLE-2006-BLG-265 with the RV standard HD $222563\left(\mathrm{RV}=5.6 \mathrm{~km} \mathrm{~s}^{-1}\right.$; Udry et al. 1999), we found a heliocentric RV of $-154 \mathrm{~km} \mathrm{~s}^{-1}$ for the microlensed star. This measurement, together with the source location projected toward the dense fields of the bulge, supports its identification as a bulge star.

The spectrum clearly shows features consistent with its high reddening and large distance from the Sun, particularly the interstellar absorption around the $\mathrm{Na} \mathrm{D}$ lines and the presence of the strong diffuse interstellar bands at 5780 and $6283 \AA$ (Herbig 1995). In Figure 1 we show part of the spectrum.

\section{ABUNDANCE ANALYSIS}

We measured equivalent widths with SPECTRE, ${ }^{13}$ and then translated those into abundances with the 2002 version of MOOG $^{14}$ (Sneden 1973). We interpolated the Kurucz grid GRIDP $05^{15}$ with overshooting for $[\mathrm{M} / \mathrm{H}]=0.5$ (the limit of the Kurucz grid) for our model atmosphere. We found the $3 \sigma$ limit for Li by performing a $\chi^{2}$ test with the data and synthetic spectra from MOOG.

\subsection{Model Atmosphere Parameters}

We set $T_{\text {eff }}$ by requiring that there be no trend in derived abundance in the Fe I lines with excitation potential (EP). We next adopted the $\log g$ for which the average Fe abundance from the Fe I and the Fe II lines was the same to within 0.01 dex.

We derived the microturbulent velocity $\xi$ by demanding no correlation between the $\mathrm{Fe}$ I abundance derived from a line and its equivalent width. Our final values are $T_{\text {eff }}=5650 \pm 150 \mathrm{~K}$, $\log g=4.4 \pm 0.3, \quad \xi=1.2 \pm 0.3 \mathrm{~km} \mathrm{~s}^{-1}$ and $[\mathrm{M} / \mathrm{H}]=$ $0.50 \pm 0.15$, where the error in $\log g$ is essentially all due to uncertainty in $T_{\text {eff }}$.

To check our parameters, we combined the spectroscopic $T_{\text {eff }}$ with the microlens-estimated radius to find the luminosity and then converted this to mass using the zero-age main-sequence

\footnotetext{
12 IRAF is distributed by the National Optical Astronomy Observatories, which are operated by the Association of Universities for Research in Astronomy, Inc., under cooperative agreement with the National Science Foundation.

${ }^{13} \mathrm{See}$ http://verdi.as.utexas.edu/spectre.html.

${ }^{14}$ See http://verdi.as.utexas.edu/moog.html.

${ }^{15}$ See http://kurucz.harvard.edu/grids.html.
} 
TABLE 1

Equivalent WidthS AND LiNe INFORMation

\begin{tabular}{ccccccc}
\hline \hline Ion & $\begin{array}{c}\lambda \\
(\AA)\end{array}$ & $\begin{array}{c}\mathrm{EP} \\
(\mathrm{eV})\end{array}$ & $\log g f$ & $\begin{array}{c}\text { Damping }^{\mathrm{a}} \\
\text { Factor }\end{array}$ & $\begin{array}{c}\mathrm{EW}^{\mathrm{b}} \\
(\mathrm{m} \AA)\end{array}$ & Isotope \\
\hline Ba II $\ldots . .$. & 5853.685 & 0.600 & -2.068 & 1.0 & 74.0 & 135 \\
& 5853.685 & 0.600 & -2.068 & 1.0 & $\mathrm{~b}$ & 137 \\
& 5853.686 & 0.600 & -2.010 & 1.0 & $\mathrm{~b}$ & 137 \\
& 5853.686 & 0.600 & -2.213 & 1.0 & $\mathrm{~b}$ & 137 \\
\hline
\end{tabular}

Note. - Table 1 is published in its entirety in the electronic edition of the Astrophysical Journal. A portion is shown here for guidance regarding its form and content.

${ }^{\text {a }}$ Correction factor to the Unsöld approximation.

$\mathrm{b}$ "b" is treated as a blend.

Padua isochrones for $Z=0.030$ (Girardi et al. 2002). This yielded $\log g=4.34$, in good agreement with our spectroscopic value. We also converted the spectroscopic $T_{\text {eff }}$ to color (Alonso et al. 1996), which yielded $(V-I)_{0}=0.705 \pm$ 0.04 , i.e., within $1.2 \sigma$ of the microlens-estimated value.

\subsection{Atomic Data}

We adopted the line lists from Bensby et al. (2003, 2004) for almost all lines. The exceptions are the Mn I, Sc II, V I, $\mathrm{Co}$ I, and $\mathrm{Ba}$ II, and some $\mathrm{Cr}$ I lines, for which we used the Vienna Atomic Line Database (VALD; Kupka et al. 2000) values. We used the hyperfine splitting line lists for $\mathrm{Mn}, \mathrm{V}, \mathrm{Sc}$, $\mathrm{Co}, \mathrm{Cu}$, and $\mathrm{Ba}$ from Johnson et al. (2006), and references therein. We adopted the solar system isotopic ratios for the $\mathrm{Cu}$ and $\mathrm{Ba}$ isotopes. The enhancement factors to the Unsöld (1955) damping constants from both Bensby et al. papers were used for elements they studied. Otherwise, the Unsöld values were adopted. The non-local thermodynamic equilibrium (non-LTE) corrections of the $\mathrm{O}$ I triplet were taken from Bensby et al. (2004). Finally, we used the photospheric abundances of Grevesse \& Sauval (1998) as the solar values. Table 1 lists the equivalent widths and atomic parameters for the lines measured.

\subsection{Error Analysis}

We calculated errors for both the $\log \epsilon(\mathrm{X}) \equiv \log \left(N_{\mathrm{X}} / N_{\mathrm{H}}\right)+$ 12.0 and $[\mathrm{X} / \mathrm{Fe}]$ values using the general method described in McWilliam et al. (1995). Correlations between $T_{\text {eff }}$ and $\log g$, $[\mathrm{M} / \mathrm{H}]$ and $T_{\text {eff }}, \log g$ and $\xi$ were taken into account, while the
TABLE 2

ABUNDANCES

\begin{tabular}{|c|c|c|c|c|c|c|}
\hline \multirow[b]{2}{*}{ ION } & \multirow{2}{*}{$\begin{array}{c}\log \epsilon \\
(\sigma)\end{array}$} & \multirow{2}{*}{$\begin{array}{c}{[\mathrm{X} / \mathrm{Fe}]} \\
(\sigma)\end{array}$} & \multirow[b]{2}{*}{$\sigma$} & \multirow[b]{2}{*}{ LINES } & \multicolumn{2}{|c|}{ SOlar } \\
\hline & & & & & Measured & Adopted \\
\hline $\mathrm{O}_{\mathrm{I}}$. & $8.86(10)$ & $-0.59(18)$ & 0.10 & 3 & 8.91 & 8.83 \\
\hline $\mathrm{Na} \mathrm{I}$ & $6.95(11)$ & $+0.06(14)$ & 0.19 & 4 & 6.22 & 6.33 \\
\hline Mg I . & $8.06(19)$ & $-0.08(19)$ & 0.17 & 1 & 7.73 & 7.58 \\
\hline $\mathrm{Al} \mathrm{I} \ldots \ldots$ & $7.28(16)$ & $+0.25(16)$ & 0.20 & 2 & 6.51 & 6.47 \\
\hline Si I $\ldots \ldots$. & $8.08(07)$ & $-0.03(10)$ & 0.16 & 14 & 7.57 & 7.55 \\
\hline$S_{\text {I }} \ldots \ldots \ldots$ & $7.61(36)$ & $-0.28(39)$ & 0.36 & 1 & 7.19 & 7.33 \\
\hline $\mathrm{Ca} \mathrm{I} \ldots \ldots \ldots$ & $6.69(11)$ & $-0.23(09)$ & 0.22 & 12 & 6.30 & 6.35 \\
\hline Sc II $\ldots \ldots$ & $3.90(21)$ & $+0.17(12)$ & 0.19 & 4 & 3.23 & 3.17 \\
\hline Ti I & $5.48(19)$ & $-0.10(10)$ & 0.14 & 6 & 4.89 & 4.99 \\
\hline$V_{\text {I }} \ldots \ldots \ldots$ & 4.61(11) & $+0.05(13)$ & 0.15 & 8 & 3.87 & 4.00 \\
\hline Cr I $\ldots \ldots$ & $6.31(13)$ & $+0.08(07)$ & 0.21 & 14 & 5.70 & 5.67 \\
\hline Mn I & $5.96(17)$ & $+0.01(10)$ & 0.09 & 6 & 5.33 & 5.39 \\
\hline $\mathrm{Fe} \mathrm{I} \ldots \ldots$ & $8.05(14)$ & $-0.01(\ldots)$ & 0.19 & 98 & 7.50 & 7.50 \\
\hline Fe II $\ldots$. & $8.07(16)$ & $+0.01(07)$ & 0.16 & 9 & 7.58 & 7.50 \\
\hline Co I $\ldots \ldots$ & $5.54(25)$ & $+0.06(24)$ & 0.22 & 1 & 4.81 & 4.92 \\
\hline Ni I $\ldots \ldots$. & $6.87(15)$ & $+0.06(04)$ & 0.18 & 31 & 6.20 & 6.25 \\
\hline $\mathrm{Cu}$ I $\ldots \ldots$ & $5.04(34)$ & $+0.27(27)$ & 0.25 & 1 & 4.15 & 4.21 \\
\hline Ba II . & $2.66(27)$ & $-0.03(15)$ & 0.08 & 3 & 2.39 & 2.13 \\
\hline
\end{tabular}

correlations of other combinations of parameters are negligible in our analysis. We found the random error in our analysis due to uncertainties in equivalent widths and atomic data by measuring the standard error of the sample $(\sigma)$ for elements with multiple lines and dividing by the square root of the number of lines. For elements with one or two lines, we calculated the error in the equivalent width from the formula in Johnson et al. (2006) and adopted a generous error in the oscillator strength of 0.1 dex for the random error.

\section{RESULTS}

The abundances and their errors $\left(\sigma_{\epsilon}\right.$ and $\left.\sigma_{[\mathrm{X} / \mathrm{Fe}]}\right)$ are summarized in Table 2. We did an analysis with the same line list using the solar atlas of Kurucz et al. (1984) to measure equivalent widths and interpolating a model atmosphere on the same Kurucz grid. The model parameters were $T_{\text {eff }}=5770 \mathrm{~K}, \log g=4.44$ dex, $[\mathrm{M} / \mathrm{H}]=0.00$ dex and $\xi=1.15 \mathrm{~km} \mathrm{~s}^{-1}$. The measured solar values are listed in Table 2, as are the Grevesse \& Sauval (1998) values we adopted. In Figure 2, we plot the abundances compared with recent literature values for several populations in the Milky
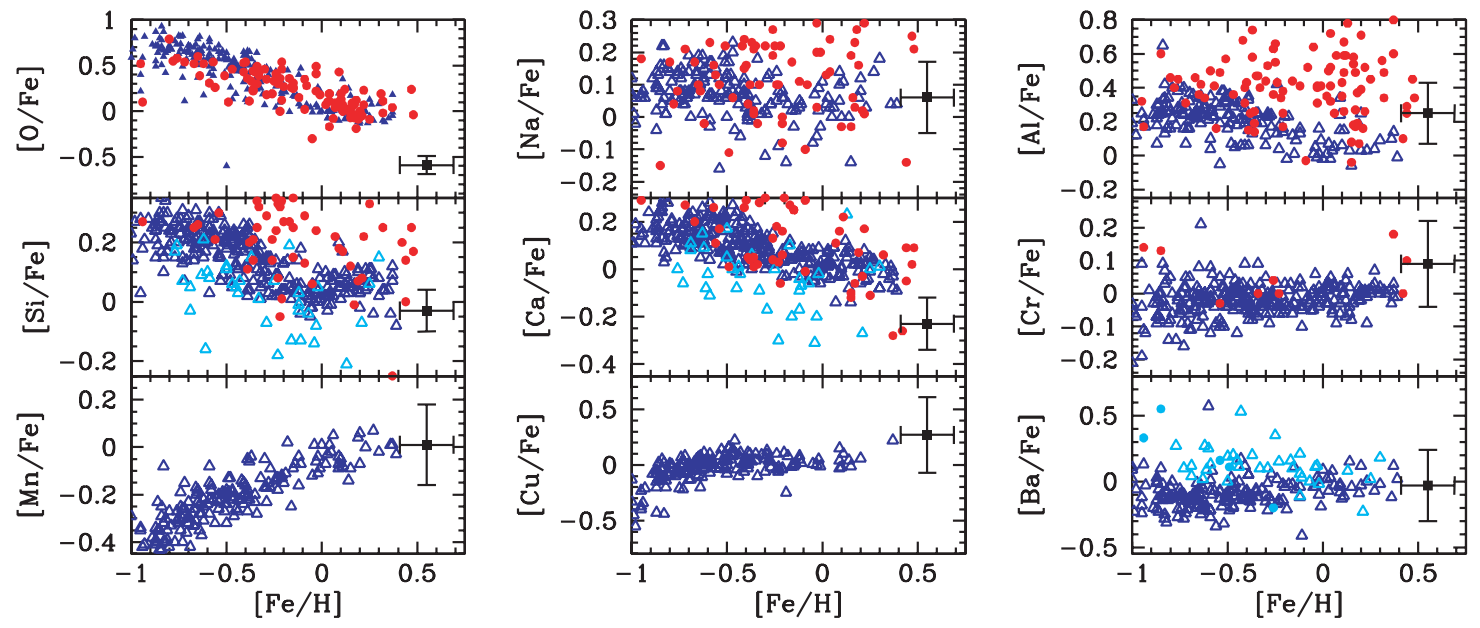

FIG. 2.-Abundance ratios for OGLE-2006-BLG-265 (black symbols) compared with local samples of thin and thick disk stars from Bensby et al. (2003, 2004, 2005), Reddy et al. (2003, 2006), and Chen et al. (2003) (blue symbols); bulge giants from McWilliam \& Rich (1994), Rich \& Origlia (2005), Fulbright et al. (2006b), and Lecureur et al. (2006) (red symbols); and "bulgelike" nearby stars from Pompéia et al. (2002, 2003) (cyan symbols). 
Way. We note three overall patterns. First, OGLE-2006-BLG265 is potentially the most metal-rich star known, although first a careful differential analysis with other extremely metal-rich stars from the bulge (Fulbright et al. 2006a) and from the local neighborhood (e.g., Santos et al. 2005) would need to be done. The high metallicity of this particular star is likely to due to chance, because the six stars from Cavallo et al. (2003) range from -1.13 to solar. Second, element ratios with $\mathrm{O}, \mathrm{Si}, \mathrm{Ca}, \mathrm{Mn}$, $\mathrm{Cu}$, and $\mathrm{Ba}$ follow the trends seen in the local disk. The $[\mathrm{O} / \mathrm{Fe}]$ and $[\mathrm{Si} / \mathrm{Fe}]$ values are lower in this star than for bulge giants of similar metallicity, according to the recent work of Fulbright et al. (2006b) and Lecureur et al. (2006). It is possible this reflects the more advanced chemical evolution recorded in the atmosphere of this main-sequence star compared to possibly older red giants or the location of the lensed dwarf in a different part of the bulge. Third, the light elements $\mathrm{Na}$ and $\mathrm{Al}$ are similar in this dwarf to the bulge giants measured by McWilliam \& Rich (1994), with the exception of the large Al enhancements seen in a few giants. The low $[\mathrm{O} / \mathrm{Fe}]$ abundance measured in the lensed dwarf is a reflection of its initial values of these elements and is clearly not the result of internal processing, as could be the case with giants. We also measured a $3 \sigma$ upper limit for $\mathrm{Li}$ of $\log \epsilon<2.08$ dex.

For most elements, the abundance ratios we observe in OGLE2006-BLG-265 are expected for a star of its metallicity if we extrapolate the results of the canonical chemical evolution models of Timmes et al. (1995). In their scenario, the low $[\mathrm{O} / \mathrm{Fe}]$, $[\mathrm{Mg} / \mathrm{Fe}],[\mathrm{Si} / \mathrm{Fe}],[\mathrm{Ca} / \mathrm{Fe}]$, and $[\mathrm{Ti} / \mathrm{Fe}]$ (and possibly $[\mathrm{S} / \mathrm{Fe}]$ ) ratios reflect the increasing importance of Fe contributed by Type Ia supernovae, while the solar or supersolar $[\mathrm{Na} / \mathrm{Fe}],[\mathrm{Al} / \mathrm{Fe}],[\mathrm{Mn} /$ $\mathrm{Fe}]$, and $[\mathrm{Cu} / \mathrm{Fe}]$ (and possibly $[\mathrm{Co} / \mathrm{Fe}]$ ) are the result of the greater ease of forming these odd-Z elements in more metal-rich Type II SNe. The solar value of $[\mathrm{Ba} / \mathrm{Fe}]$ is expected as the $s$ process contribution from asymptotic giant branch (AGB) stars keeps up with the Fe production. All of these conclusions suggest that for this bulge star at $[\mathrm{Fe} / \mathrm{H}]=+0.56 \mathrm{dex}$, the enrichment history has lasted a considerable period, longer than for the bulge stars with high $[\alpha / \mathrm{Fe}]$ values. The ratio of the iron-peak elements $\mathrm{Sc}, \mathrm{V}, \mathrm{Cr}$, and $\mathrm{Ni}$ to $\mathrm{Fe}$ are more unexpected, being elevated in all cases above the solar value, although by small amounts. Additional stars in the bulge, whether giants or dwarfs, will be helpful in determining the robustness of those results. Possible enhancements in the iron-peak elements have been seen in Galactic populations such as in Sc, V, and Co in the thick disk (Prochaska et al. 2000) and $\mathrm{Cr}$ in the bulge (McWilliam \& Rich 1994). The interpretation of $\mathrm{Li}$ abundances is complicated by internal destruction in stars before joining the main sequence. Our upper limit is near the level of Li measured in stars of similar temperature in the Hyades, a young cluster, and higher than the upper limits determined for older stars in M67 and in the Galactic disk (Balachandran 1995; Lambert \& Reddy 2004).

This Letter shows that unique information can be obtained by taking advantage of the magnification from microlensing to observe otherwise unattainable objects. With a dozen spectra per year of bulge dwarf stars, selected without any abundance bias, we have the opportunity to study the stellar populations in the bulge in a new way.

We acknowledge support from Hubble Fellowship HST-HF01158.01-A (NASA contract NAS-5-2655; A. G.-Y.); NSF AST-042758, NASA NNG04GL51G (A. G.); NASA HST-AR10673 (NASA contract NAS5-26555; D. C. L.); NSF AST0204908, NASA grant NAG5-12212, Polish MNiSW BST grant (A. U.); We recognize and acknowledge the very significant cultural role and reverence that the summit of Mauna Kea has always had within the indigenous Hawaiian community. We are most fortunate to have the opportunity to conduct observations from this mountain.

\section{REFERENCES}

Alonso, A., Arribas, S., \& Martinez-Roger, C. 1996, A\&A, 313, 873

Balachandran, S. 1995, ApJ, 446, 203

Barlow, T. 2002, MAKEE Keck Observatory HIRES Data Reduction Software

(Pasadena: Caltech), http://spider.ipac.caltech.edu/staff/tab/makee/index.html

Benetti, S., Pasquini, L., \& West, R. M. 1995, A\&A, 294, L37

Bensby, T., Feltzing, S., \& Lundström, I. 2003, A\&A, 410, 527 2004, A\&A, 415, 155

Bensby, T., Feltzing, S., Lundström, I., \& Ilyin, I. 2005, A\&A, 433, 185

Boesgaard, A. M., Deliyannis, C. P., Stephens, A., \& King, J. R. 1998, ApJ, 493, 206

Cavallo, R. M., Cook, K. H., Minniti, D., \& Vandehei, T. 2003, Proc. SPIE, 4834, 66

Chen, Y. Q., Zhao, G., Nissen, P. E., Bai, G. S., \& Qiu, H. M. 2003, ApJ, 591,925

Dong, S., et al. 2006, ApJ, 642, 842

Einstein, A. 1936, Science, 84, 506

Fulbright, J. P., McWilliam, A., \& Rich, R. M. 2006a, ApJ, 636, 821 2006b, ApJ, 636, 821

Girardi, L., Bertelli, G., Bressan, A., Chiosi, C., Groenewegen, M. A. T., Marigo, P., Salasnich, B., \& Weiss, A. 2002, A\&A, 391, 195

Gould, A., et al. 2006, ApJ, 644, L37

Grevesse, N., \& Sauval, A. J. 1998, Space Sci. Rev., 85, 161

Griest, K., \& Safizadeh, N. 1998, ApJ, 500, 37

Herbig, G. H. 1995, ARA\&A, 33, 19

Johnson, J. A., Ivans, I. I., \& Stetson, P. B. 2006, ApJ, 640, 801

Kupka, F. G., Ryabchikova, T. A., Piskunov, N. E., Stempels, H. C., \& Weiss, W. W. 2000, Baltic Astron., 9, 59

Kurucz, R. L., Furenlid, I., Brault, J., \& Testerman, L. 1984, National Solar Observatory Atlas (Sunspot: NSO)
Lambert, D. L., \& Reddy, B. E. 2004, MNRAS, 349, 757

Lecureur, A., Hill, V., Zoccali, M., Barbuy, B., Gomez, A., Minniti, D., Ortolani, S., \& Renzini, A. 2006, A\&A, in press (astro-ph/0610346)

Mao, S., \& Paczyński, B. 1991, ApJ, 374, L37

McWilliam, A., Preston, G. W., Sneden, C., \& Searle, L. 1995, AJ, 109, 2757

McWilliam, A., \& Rich, R. M. 1994, ApJS, 91, 749

Minniti, D., Vandehei, T., Cook, K. H., Griest, K., \& Alcock, C. 1998, ApJ, 499, L175

Pompéia, L., Barbuy, B., \& Grenon, M. 2002, ApJ, 566, 845 . 2003, ApJ, 592, 1173

Prochaska, J. X., Naumov, S. O., Carney, B. W., McWilliam, A., \& Wolfe, A. M. 2000, AJ, 120, 2513

Reddy, B. E., Lambert, D. L., \& Allende Prieto, C. 2006, MNRAS, 367, 1329

Reddy, B. E., Tomkin, J., Lambert, D. L., \& Allende Prieto, C. 2003, MNRAS, 340,304

Rich, R. M., \& Origlia, L. 2005, ApJ, 634, 1293

Santos, N. C., Israelian, G., Mayor, M., Bento, J. P., Almeida, P. C., Sousa, S. G., \& Ecuvillon, A. 2005, A\&A, 437, 1127

Sneden, C. A. 1973, Ph.D. thesis, Univ. Texas, Austin

Timmes, F. X., Woosley, S. E., \& Weaver, T. A. 1995, ApJS, 98, 617

Udalski, A. 2003, Acta Astron., 53, 291

Udalski, A., et al. 2005, ApJ, 628, L109

Udry, S., et al. 1999, in ASP Conf. Ser. 185, Precise Stellar Radial Velocities, ed. J. B. Hearnshaw \& C. D. Scarfe (San Francisco: ASP), 383

Unsöld, A. 1955, Physik der Sternatmosphären (Berlin: Springer)

Vogt, S. S., et al. 1994, Proc. SPIE, 2198, 362

Yoo, J., et al. 2004, ApJ, 603, 139 